\title{
Emergency department overcrowding, mortality and the 4-hour rule in Western Australia
}

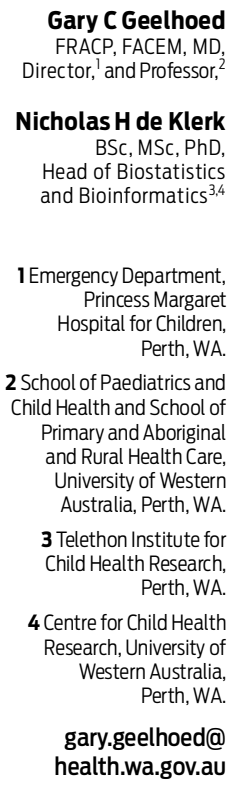

MJA 2012; 196: 122-126 doi: 10.5694/mjall.11159

Editorial p 88 Commentary $p 126$ Research p 128 vercrowding in emergency departments (EDs) occurs when the physical or staffing capacity of the department is exceeded by the number of patients waiting to be seen, undergoing assessment and treatment or waiting for departure. ${ }^{1}$ Overcrowding occurs in Australia and elsewhere in the developed world and has been well documented over the past decade. ${ }^{2-4}$

It became generally recognised that this problem had come about through a combination of factors. Upstream factors included increased numbers of patients presenting to emergency departments, a rapidly growing population and reduction of available public hospital beds. ${ }^{5}$ Downstream factors included lack of aged care beds in the community. ${ }^{6}$ It is generally accepted that overcrowding in Australia and other countries is due to bed occupancy rates above $85 \%$ rather than increased presentation of low acuity patients. ${ }^{7-11}$

About a decade ago, Australian health authorities introduced initiatives aimed at coping with the increasing demand. Task forces developed strategies that included improving efficiency and monitoring inpatients who stayed for longer periods of time. ${ }^{12-14}$ In 2008, the Western Australian government announced that EDs would adopt a 4-hour rule, whereby firstly $85 \%$ and eventually $98 \%$ of patients would be either discharged home or admitted to a ward within 4 hours of presentation. Following a planning phase, the 4-hour rule came into effect in three tertiary hospitals in Perth, the capital city, in October 2009. One year later, the 4hour rule was introduced at three secondary hospitals in Perth. As of June 2010, Western Australia had a population of 2.3 million people, of whom $77 \%$ resided in Perth. ${ }^{15}$

\begin{abstract}
Objective: To assess whether emergency department (ED) overcrowding was reduced after the introduction of the 4-hour rule in Western Australia and whether any changes in overcrowding were associated with significant changes in patient mortality rates.
\end{abstract}

Design, setting and patients: Quasi-experimental intervention study using dependent pretest and post-test samples. Hospital and patient data were obtained for three tertiary hospitals and three secondary hospitals in Perth, WA, for 2007-08 to 2010-11.

Main outcome measures: Mortality rates; overcrowding rates.

Results: No change was shown in mortality from 2007-08 to 2010-11 for the secondary hospitals and from 2007-08 to 2009-10 for the tertiary hospitals. ED overcrowding (as measured by 8-hour access block) at the tertiary hospitals improved dramatically, falling from above $40 \%$ in July 2009 to around $10 \%$ by early 2011 , and presentations increased by $10 \%$, while the mortality rate fell significantly (by $13 \%$; $95 \% \mathrm{Cl}, 7 \%-18 \% ; P<0.001$ ) from $1.12 \%$ to $0.98 \%$ between 2009-10 and 2010-11. Monthly mortality rates decreased significantly in two of the three tertiary hospitals concurrently with decreased access block and an increased proportion of patients admitted in under 4 hours.

Conclusion: Introduction of the 4-hour rule in WA led to a reversal of overcrowding in three tertiary hospital EDs that coincided with a significant fall in the overall mortality rate in tertiary hospital data combined and in two of the three individual hospitals. No reduction in adjusted mortality rates was shown in three secondary hospitals where the improvement in overcrowding was minimal.

We aimed to establish whether the yearly mortality rate for patients presenting to these hospitals changed significantly after the 4-hour rule was introduced. We hypothesised that any decrease in overcrowding would be reflected in a decrease in mortality rate. A secondary objective was to examine the association between two measures of overcrowding and monthly mortality rates for patients presenting to each of the six EDs from July 2009 to June 2011.

\section{Methods}

This study was designed as a quasiexperimental intervention study using dependent pretest and post-test samples. In this study, access block is defined as the percentage of patients requiring admission who wait more than 8 hours from presentation for an inpatient bed.

\section{Study setting}

The study included Perth's three tertiary adult hospitals (Hospitals 1-3) and three of four secondary hospitals (Hospitals 4-6). Hospitals 1 and 2 treat only adults; 3-6 treat adults and children.

\section{Data collection}

Yearly data were obtained from the WA Health Department on access block at Hospitals 1-6 for 2000-2008 and on presentations, admissions and deaths for 2007-08 and 2008-09. We also obtained monthly data for July 2009 to June 2011, which included the number of patients presenting to each of the hospitals; the number admitted; the number of deaths among those admitted through the EDs; the number of admitted patients who gained access to an inpatient bed within 4 hours; and the number of patients who could not gain access to 
1 Characteristics of three Perth tertiary hospitals, July 2007 to June 2011

\begin{tabular}{|c|c|c|c|c|c|c|}
\hline & Hospital 1 & Hospital 2 & Hospital 3 & Total & Rate difference* $(95 \% \mathrm{Cl})$ & Rate ratio ${ }^{\dagger}(95 \% \mathrm{Cl})$ \\
\hline \multicolumn{7}{|l|}{ 2007-08 } \\
\hline Presentations & 51035 & 57944 & 48404 & 157383 & & \\
\hline Admissions & 22371 & 23842 & 16066 & 62279 & & \\
\hline Deaths (mortality rate) & $689(1.35 \%)$ & $626(1.08 \%)$ & $419(0.87 \%)$ & $1734(1.10 \%)$ & - & - \\
\hline \multicolumn{7}{|l|}{ 2008-09 } \\
\hline Presentations & 52476 & 60760 & 46746 & 159982 & & \\
\hline Admissions & 24791 & 25808 & 16053 & 66652 & & \\
\hline Deaths (mortality rate) & $674(1.28 \%)$ & $684(1.13 \%)$ & $439(0.94 \%)$ & $1797(1.12 \%)$ & $0.02(-0.05$ to 0.09$)$ & $2.0 \%$ ( -4.6 to 8.9$)$ \\
\hline \multicolumn{7}{|l|}{ 2009-10 } \\
\hline Presentations & 56115 & 64215 & 48434 & 168764 & & \\
\hline Admissions & 29127 & 28248 & 19691 & 77066 & & \\
\hline Deaths (mortality rate) & $698(1.24 \%)$ & $670(1.04 \%)$ & $528(1.09 \%)$ & $1896(1.12 \%)$ & $0.00(-0.07$ to 0.07$)$ & 0.0 (- 6.3 to 6.7$)$ \\
\hline \multicolumn{7}{|l|}{ 2010-11 } \\
\hline Presentations & 60455 & 71091 & 53947 & 185493 & & \\
\hline Admissions & 33661 & 33997 & 23388 & 91046 & & \\
\hline Deaths (mortality rate) & $710(1.17 \%)$ & $633(0.89 \%)$ & $473(0.88 \%)$ & $1816(0.98 \%)$ & $-0.14(-0.21$ to -0.08$)$ & $-12.9 \%(-18.3$ to -7.1$)$ \\
\hline
\end{tabular}

2 Characteristics of three Perth secondary hospitals, July 2007 to June 2011

\begin{tabular}{|c|c|c|c|c|c|c|}
\hline & Hospital 4 & Hospital 5 & Hospital 6 & Total & Rate difference* $(95 \% \mathrm{Cl})$ & Rate ratio ${ }^{\dagger}(95 \% \mathrm{Cl})$ \\
\hline \multicolumn{7}{|l|}{$2007-08$} \\
\hline Presentations & 45739 & 43665 & 33277 & 122681 & & \\
\hline Admissions & 4623 & 7719 & 4413 & 16755 & & \\
\hline Deaths (mortality rate) & $77(0.17 \%)$ & $110(0.25 \%)$ & $38(0.11 \%)$ & $225(0.18 \%)$ & - & - \\
\hline \multicolumn{7}{|l|}{ 2008-09 } \\
\hline Presentations & 43578 & 43533 & 34445 & 121556 & & \\
\hline Admissions & 4859 & 9077 & 5613 & 19549 & & \\
\hline Deaths (mortality rate) & $72(0.17 \%)$ & $120(0.28 \%)$ & $63(0.18 \%)$ & $255(0.21 \%)$ & 0.03 (- 0.01 to 0.06$)$ & $14.4 \%$ ( -4.4 to 36.8 ) \\
\hline \multicolumn{7}{|l|}{ 2009-10 } \\
\hline Presentations & 46258 & 46909 & 36834 & 130001 & & \\
\hline Admissions & 5539 & 10382 & 6636 & 22557 & & \\
\hline Deaths (mortality rate) & $82(0.18 \%)$ & $100(0.21 \%)$ & $69(0.19 \%)$ & $251(0.19 \%)$ & $-0.02(-0.05$ to 0.02$)$ & $-8.0 \%(-22.7$ to 9.6$)$ \\
\hline \multicolumn{7}{|l|}{ 2010-11 } \\
\hline Presentations & 46978 & 51293 & 40118 & 138389 & & \\
\hline Admissions & 6900 & 10626 & 8384 & 25910 & & \\
\hline Deaths (mortality rate) & $76(0.16 \%)$ & $122(0.24 \%)$ & $60(0.15 \%)$ & $258(0.19 \%)$ & $-0.01(-0.04$ to 0.03$)$ & $-3.5 \%(-18.8$ to 14.9$)$ \\
\hline
\end{tabular}

an inpatient bed by 8 hours. Data on ED activity and on mortality were extracted from WA's Emergency Department Data Collection and Hospital Morbidity Data Collection, respectively.

\section{Statistical methods}

For all hospitals, yearly mortality rates were calculated using the numbers of patients admitted from the ED who died during their episode of care, with denominators of the number of presentations, to allow for the increase in the percentage of patients who were admitted during the study period. $\chi^{2}$ analysis was used to compare the proportions of patients who died in the hospitals from 2007-08 to 2010-11. Poisson regression was used to examine the relationship between monthly mortality rates from July 2009 to June 2011 for the six hospitals, the percentage of admitted patients who gained access to an inpatient bed within 4 hours, and the percentage of patients who could not access an inpatient bed by 8 hours, adjusting for effects of individual months (for seasonal effects), and individual hospitals when analyses were grouped into tertiary or secondary hospitals. Some models included a single overall trend variable, coded 1 to 24 for the months of the study, to estimate the average monthly increase or decline over the whole 2year period. Rate ratios were estimated per $10 \%$ change in overcrowding measure. Yearly comparisons between mortality rates were done using Poisson regression to estimate rate ratios and binomial regression to estimate rate differences. Microsoft Excel 2003 (Microsoft Corporation, 
3 Yearly access block* at six Perth hospitals, 2000 to 2008

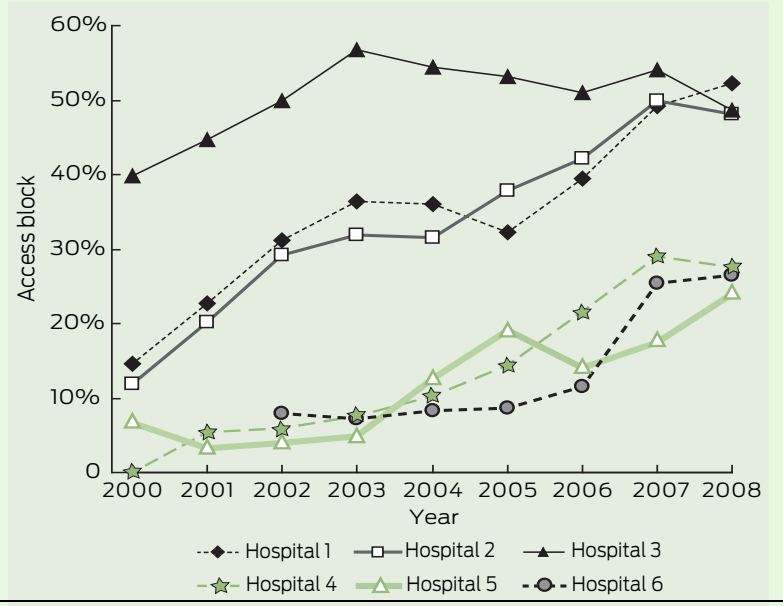

$\mathrm{H} 1-\mathrm{H} 3$ are tertiary hospitals. $\mathrm{H} 4-\mathrm{H} 6$ are secondary hospitals. * Percentage of patients requiring admission who wait more than 8 hours from presentation for an inpatient bed.

4 Monthly access block* at three Perth tertiary hospitals, July 2009 to June 2011

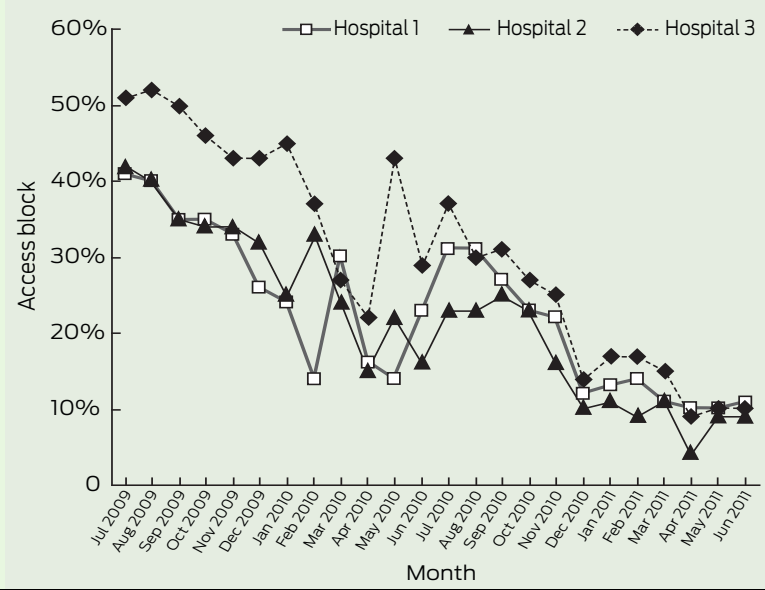

* Percentage of patients requiring admission who wait more than 8 hours from presentation for an inpatient bed.

5 Monthly access block* at three Perth secondary hospitals, July 2009 to June 2011

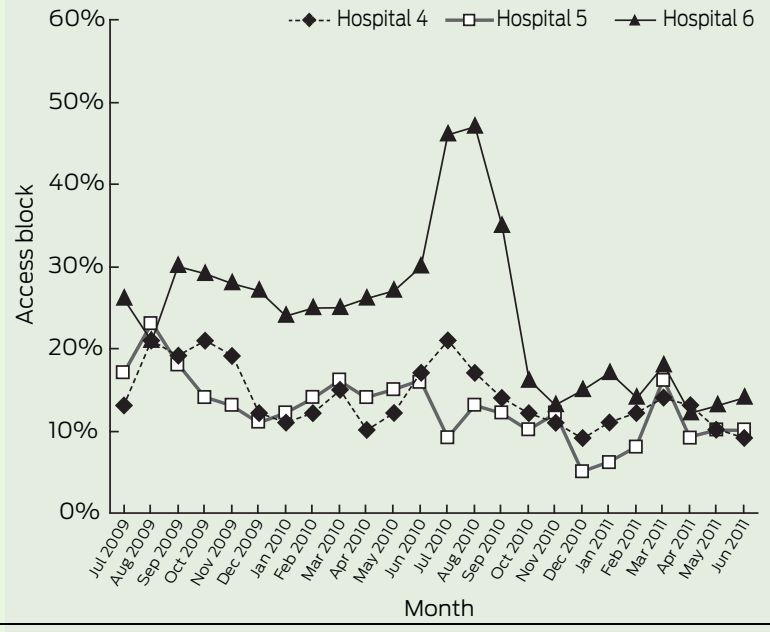

* Percentage of patients requiring admission who wait more than 8 hours from presentation for an inpatient bed.
Redmond, Wash, USA), SPSS, version 19 (SPSS Inc, Chicago, Ill, USA) and Stata, version 10.2 (StataCorp, College Station, Tex, USA) were used for statistical analysis.

\section{Ethics approval}

Ethics approval was sought from the Human Research Ethics Committee of the WA Health Department, which considered it unnecessary, given the de-identified nature of the data.

\section{Results}

The numbers of patients presenting to the EDs and the percentages of patients admitted increased during the study period (Box 1 and Box 2).

Overcrowding, as measured by access block, rose throughout 20002008 at the six hospitals (Box 3). Monthly access block in the tertiary hospitals fell from above $40 \%$ in July 2009 to around 10\% by early 2011 (Box 4), while the three secondary hospitals, at a lower level overall, showed a much smaller decrease (Box 5).

No change was seen in mortality from 2007-08 to 2009-10 at the tertiary hospitals (Box 1). From 2009-10 to 2010-11, at the same time as ED overcrowding decreased dramatically (Box $4)$, presentations increased by $10 \%$ while the overall relative mortality rate fell significantly, by $13 \%$ ( $95 \% \mathrm{CI}$, $7 \%-18 \% ; P<0.001)$, an absolute rate reduction of $0.14 \%$. The relative mortality rates, and the absolute rate reductions for Hospitals 2 and 3 were similar for the last two periods, while Hospital 1 had less decline. No significant changes were seen in the overall mortality rate from 2007-08 to 201011 for the secondary hospitals (Box 2). Monthly mortality rates for all hospitals for the 24 months from July 2009 to June 2011 were compared with measures of ED overcrowding, and grouped relative mortality rates are shown in Box 6. After adjusting for monthly or seasonal effects, there was a significant decline in the mortality rate from July 2009 to June 2011 in the tertiary hospitals. There was a decrease in mortality rate associated with decreased access block or with an increase in the proportion of patients who gained access to an inpatient bed in less than 4 hours. When both the linear trend and either of the overcrowding terms were included in the same model, neither was significant and the overall trend was reduced to near zero. However, for access block, even after adjusting for the overall time trend, the estimated reduction was still $8.0 \%$ per 10 percentage point decrease $(95 \% \mathrm{CI}$, -0.2 to $17.5 ; P=0.07$ ). In individual Poisson models for each of the six hospitals, monthly access block was a more significant predictor of mortality than the linear time trend. There was some evidence of heterogeneity of monthly decline $(P=0.03)$ within the three tertiary hospitals, with a smaller monthly decline in Hospital 1. There was, however, no significant heterogeneity in the associations between mortality and overcrowding measures.

\section{Discussion}

This study demonstrates that improvement in ED overcrowding after the introduction of the 4-hour rule in three tertiary hospitals in WA was associated with fewer deaths and a reduced mortality rate. Concurrently, three secondary hospitals that did not experience significant improvement in overcrowding experienced no significant reduction in mortality rates.

Evidence suggests that overcrowding leads to poor health outcomes. Increased risk of recurrent myocardial infarction has been shown to be associated with prolonged ED stays. ${ }^{16} \mathrm{~A}$ relative risk of death at 10 days of 1.34 has been shown among patients presenting during periods of ED overcrowding compared with periods when they were not overcrowded. Most of these patients were classified as more urgent and had a higher mortality rate by triage category ${ }^{17} \mathrm{~A}$ raised incidence rate ratio during ambulance diversion, compared with a non-diversion period, has been reported. ${ }^{18}$ A Victorian study found that the average excess length of stay for inpatients ranged from 0.39 days among patients who stayed in the ED for 4 hours or less, to 2.35 days among patients who were in the ED for more than 12 hours. ${ }^{19}$ A study in WA showed that ED overcrowding in Perth's three tertiary hospitals was associated with an estimated 120 deaths in $2003 .{ }^{20}$ 
6 Adjusted* relative mortality rates based on two measures of overcrowding, and linear monthly trend, for emergency department patients at six Perth hospitals during the months July 2009 to June 2011

Relative decline in mortality rate $(95 \% \mathrm{Cl} ; P)$

\begin{tabular}{|c|c|c|}
\hline \multirow{2}{*}{ Measure of overcrowding } & \multirow{2}{*}{\multicolumn{2}{|c|}{ Three secondary hospitals }} \\
\hline & & \\
\hline $\begin{array}{l}\text { Patient gained access to an inpatient bed in }<4 \mathrm{~h} \\
\text { (per } 10 \text { percentage point increase) }\end{array}$ & $9.2 \%(4.1 \%$ to $12.1 \% ;<0.001)$ & $15.0 \%$ (-19.4\% to 39.5\%; 0.35) \\
\hline \multirow{3}{*}{$\begin{array}{l}>8 \mathrm{~h} \text { for patient to gain access to inpatient bed } \\
\text { (access block) (per } 10 \text { percentage point } \\
\text { decrease) }\end{array}$} & $9.3 \%$ (5.1\% to 13.7\%; < 0.001$)$ & $2.3 \%$ (-15.4\% to 17.4\%; 0.78) \\
\hline & \multicolumn{2}{|c|}{ Average decline in mortality per month ( $95 \% \mathrm{Cl}$; P) } \\
\hline & $1.1 \%(0.6 \%$ to $1.7 \% ;<0.001)$ & $0.3 \%(-1.2 \%$ to $1.7 \% ; 0.73)$ \\
\hline
\end{tabular}

Pressure on emergency departments in WA tertiary hospitals had increased dramatically over 10 years due to the increasing numbers presenting to the hospitals' EDs, coupled with reduced available public bed numbers and fixed nurse-patient ratios on wards. The introduction of the 4-hour rule encouraged hospitals as a whole to share the responsibility for, and help solve the problem of, overcrowding in EDs. This whole-ofhospital approach appears to have led to better communication between the EDs and the wards, with an increased appreciation of each other's problems and challenges. A revised target of $85 \%$ of patients discharged within 4 hours was achieved by the tertiary hospitals by early 2011 .

While success in reducing overcrowding in the EDs was obvious, questions were asked as to whether this change might have led to poorer patient outcomes due to rushed decisions and clinically inappropriate transfer of patients to wards. Junior medical staff, in particular, felt that the process was difficult, as they no longer initially managed many of these patients in the protected environment of the emergency department with many senior staff on hand to supervise. Increasingly, they have had to change their practice, with more admissions and investigations occurring on the wards rather than in the EDs. A survey of junior medical staff in WA also illustrated their concerns regarding reduced opportunities for training in patient safety. ${ }^{21}$

When the 4-hour rule was introduced, however, it was with the expectation of improved clinical outcomes. Before the introduction of the rule, it was estimated that Australia was about 4000 beds short of what was needed to achieve an $85 \%$ occupancy rate, ${ }^{22,23}$ which is generally accepted as being an ideal target to enable hospitals to function both safely and efficiently. Given earlier work suggesting that significantly increased mortality was associated with overcrowding in WA's emergency departments, ${ }^{20}$ the expectation that reduced overcrowding would lead to reduced mortality was reasonable. It is clear that access block rose in all six hospitals from 2000 to 2008 then started to fall before the official start of the 4-hour rule - presumably in anticipation. This can be seen to a lesser extent in the secondary hospitals, which - given the publicity surrounding the rule's introduction to the tertiary hospitals in 2009 - is not surprising, as it was known that they would be commencing the process 1 year later. While 80 fewer deaths (1 every 4 to 5 days) occurred in the tertiary hospitals in 2010-11, if the mortality rate of 2009-10 were applied to the numbers presenting in 201011 , it could be argued that 267 lives (1 every 2 days) were saved.

By breaking down the artificial barriers between overcrowding in the EDs and the rest of the hospital, this process has effectively used existing beds more efficiently. However, given the increasing demands on public hospitals, this can only be seen as a temporary measure and in no way offsets the need to provide further beds into the future. Hopefully, however, the dialogue that now exists between EDs and the hospital wards in WA will continue under the 4-hour rule, so that overcrowding will not happen again, or, at least, not exclusively in EDs. Clearly, exposure of EDs to unlimited numbers of patients while inpatient wards are protected with "quotas" and have no responsibility for overcrowding in EDs - is unsustainable, dangerous for patients and illogical.

This study has a number of strengths. Perth's isolated position as a population centre makes it ideal for this type of study. The staggered introduction of the 4-hour rule allowed comparison of mortality rates sequentially and concurrently, in the one population. The integrated nature of the WA health system allowed for consistent data collection across the six hospitals. While these findings apply to the WA health system, there is no reason to suspect that other Australian health systems should fare differently if their hospitals similarly commit to whole-of-hospital reform. While the increase in presentations after the intervention, at around 10\%, is higher than the $6 \%$ that might be expected for the combined effect of population growth and ageing of the population, mortality rates calculated in this study were a sensible outcome measure, especially as the number of deaths recorded for the tertiary hospitals actually fell between the last two years. It is possible that changes in factors other than overcrowding may have led to a fall in the number of deaths at the tertiary hospitals; however, the mortality rates for the two previous years, 2007-08 and 2008-09, were consistent, and no such dramatic reduction was seen at the same time in the three secondary hospitals. The near significance of the effect of access block even when the overall downward trend was adjusted for, and the lack of a significant fall in mortality when any measure of overcrowding was adjusted for, also indicates that decreasing overcrowding was the driving force in these changes. 
The introduction of a 4-hour rule into three Western Australian tertiary hospital EDs was followed by fewer deaths and a significant fall in mortality for patients admitted through those departments.

Competing interests: No relevant disclosures.

Received 7 Sep 2011, accepted 21 Dec 2011

1 Australasian College for Emergency Medicine. Policy document - standard terminology. Emerg Med 2002; 14: 337-340.

2 Fatovich DM, Hirsch RL. Entry overload, emergency department overcrowding, and ambulance bypass. Emerg Med J 2003; 20: 406409.

3 Schneider SM, Gallery ME, Schafermeyer R, Zwemer FL. Emergency department crowding: a point in time. Ann Emerg Med 2003; 42: 167-172.

4 Vilke GM, Brown L, Skogland P, et al. Approach to decreasing emergency department ambulance diversion hours. J Emerg Med 2004; 26: 189-192.

5 Australian Government Department of Health and Ageing. The state of our public hospitals. June 2006 report. http://www.health.gov.au/ internet/main/publishing.nsf/Content/state-ofpublic-hospitals-report.htm (accessed Jan 2012).

6 Finn JC, Flicker L, Mackenzie E, et al. Interface between residential aged care facilities and a teaching hospital emergency department in Western Australia. Med J Aust 2006; 184: 432435.
7 Sprivulis P, Grainger S, Nagree Y. Ambulance diversion is not associated with low acuity patients attending Perth metropolitan emergency departments. Emerg Med Australas 2005; 17: 11-15.

8 Canadian Association of Emergency Physicians; National Emergency Nurses Affiliation. Joint Position Statement on emergency department overcrowding. CJEM 2001; 3: 82-84.

9 Schull MJ, Kiss A, Szalai JP. The effect of lowcomplexity patients on emergency department waiting times. Ann Emerg Med 2007; 49: 257264.

10 Sprivulis P. Estimation of the general practice workload of a metropolitan teaching hospital emergency department. Emerg Med (Fremantle) 2003; 15: 32-37.

11 Vertesi L. Does the Canadian Emergency Department Triage and Acuity Scale identify nonurgent patients who can be triaged away from the emergency department? CJEM 2004; 6: 337342.

12 Cameron PA, Campbell DA. Responses to access block in Australia: Royal Melbourne Hospital. Med J Aust 2003; 178: 109-110.

13 Fatovich DM. Responses to access block in Australia: Royal Perth Hospital. Med J Aust 2003 178: 108-109.

14 Hill KN. Responses to access block in Australia: The Alfred Hospital. Med J Aust 2003; 178: 110-111.

15 Australian Bureau of Statistics. Western Australian statistical indicators, 2010. (ABS Cat. No. 1367.5.)

16 Diercks DB, Roe MT, Chen AY, et al. Prolonged emergency department stays of non-ST- segment elevation myocardial infarction patients are associated with worse adherence to the American College of Cardiology/American Heart Association guidelines for management and increased adverse events. Ann Emerg Med 2007; 50: 489-496.

17 Richardson DB. Increase in patient mortality at 10 days associated with emergency department overcrowding. Med J Aust 2006; 184: 213-216.

18 Fatovich DM. Effect of ambulance diversion on patient mortality: how access block can save your life. Med J Aust 2005; 183: 672-673.

19 Liew D, Liew D, Kennedy MP. Emergency department length of stay independently predicts excess inpatient length of stay. Med J Aust 2003; 179: 524-526.

20 Sprivulis PC, Da Silva J-A, Jacobs IG, et al. The association between hospital overcrowding and mortality among patients admitted via Western Australian emergency departments. Med J Aust 2006; 184: 208-212

21 Maor D, O'Sullivan M, Bonning M, Mitchell R. Fourhour access targets for emergency departments: potential negative implications for the work practices and clinical training of junior doctors. Emerg Med Australas 2011; 23: 782-783.

22 Australasian College for Emergency Medicine. Access block and overcrowding in emergency departments. 2004.

23 Bagust A, Place M, Posnett JW. Dynamics of bed use in accommodating emergency admissions: stochastic simulation model. BMJ 1999; 319: 155158. 